\title{
Topological nodal points in two coupled SSH chains
}

\author{
C. $\mathrm{Li}^{1}$, S. $\mathrm{Lin}^{1}$, G. Zhang ${ }^{2}$ and Z. Song冞 \\ ${ }^{1}$ School of Physics, Nankai University, Tianjin 300071, China \\ ${ }^{2}$ College of Physics and Materials Science, Tianjin Normal University, Tianjin 30038\%, China
}

\begin{abstract}
We study two coupled Su-Schrieffer-Heeger ( $\mathrm{SSH}$ ) chains system, which is shown to contain rich quantum phases associated with topological invariants protected by symmetries. In the weak coupling region, the system supports two non-trivial topological insulating phases, characterized by winding number $\mathcal{N}= \pm 1$, and two types of edge states. The boundary between the two topological phases arises from two band closing points, which exhibit topological characteristics in onedimensional $k$ space. By mapping Bloch states on a vector field in $k$ space, the band degenerate points correspond to a pair of kinks of the field, with opposite topological charges. Two topological nodal points move and merge as the inter-chain coupling strength varies. This topological invariant is protected by the translational and inversion symmetries, rather than the antiunitary operation. Furthermore, we find that when a pair of nodal points is created, a second order quantum phase transition (QPT) occurs, associating with a gap closing and spontaneously symmetry breaking. This simple model demonstrates several central concepts in the field of quantum materials and provides a theoretical connection between them.
\end{abstract}

PACS numbers: 03.65.Vf, 64.70.Tg, 11.30.Er, 71.10.Fd

\section{INTRODUCTION}

Topological gapless systems have emerged as a new frontier in the field of quantum materials [1 17$]$. As a joint of two quantum phases, topological gapless systems have band structures with band-touching points in the momentum space, where these kind of nodal points appear as topological defects of an auxiliary vector field. Then these points are unremovable due to the symmetry protection, until a pair of them meet together and annihilate. In general, the studied systems are usually 2-D and 3-D with the broken time-reversal symmetry. The central goal of this work is to understand the physics of topological gapless system from the point of view of the 1-D model. A particular advantage in working within the 1-D system is that all the parameters of this model can be easily accessed within the existing technology of coldatomic experiments [18 20]. On the other hand, we find that unlike the topological gapless systems in $2-\mathrm{D}$, the magnetic flux is not necessary for the quasi 1-D system.

In this work, we systematically study two coupled SuSchrieffer-Heeger (SSH) chains system. Comparing to SSH chain, this ladder system contains rich quantum phases associated with topological invariants protected by symmetries. In the weak coupling region, the system supports two non-trivial topological insulating phases, characterized by winding number $\mathcal{N}= \pm 1$, and two types of edge states for the open ladder. We focus on the boundary between these two quantum phases. We find that the boundary phase arises from two band closing points, which exhibit topological characteristics in the one-dimensional $k$ space. We investigate the topological feature of the boundary by mapping Bloch states

\footnotetext{
* songtc@nankai.edu.cn
}

on a vector field in $k$ space and find that, the band degenerate points correspond to a pair of kinks of the field, with opposite topological charges. As the inter-chain coupling strength varies, two topological nodal points move and merge in $k$ space. In contrast to the case of 2-D semimetal phase, the topological invariant is protected by the translational and inversion symmetries, rather than the antiunitary operation.

In conventional QPTs, quantum phases are differentiated by the symmetry [21], while topological matters are classified according to topological invariants [22, 23]. The possible connection between two regimes is studied in a class of quantum spin models [24]. In this work, we find that when a pair of nodal points is created, a second order quantum phase transition (QPT) occurs, associating with a gap closing and spontaneously translational and inversion symmetry breaking. This simple model provides an alterative example to demonstrate the coexistence two types of QPTs, revealing the connection between them.

The remainder of this paper is organized as follows. In Sec. [II we present a two coupled-SSH chain model and the quantum phase diagram, characterized by winding number and edge states. Sec. [II reveals the topological feature of nodal points. Sec. [V] demonstrates the behavior of topological gapless system in the presence of several types of perturbations. Sec. $\nabla$ devotes to the symmetries that protect the topological invariants. Finally, we present a summary and discussion in Sec. VI

\section{MODEL AND PHASE DIAGRAM}

The SSH model [25] has served as a paradigmatic example of the 1-D system supporting topological character 26]. It has an extremely simple form but well manifests the typical feature of topological insulating phase, and 
(a)

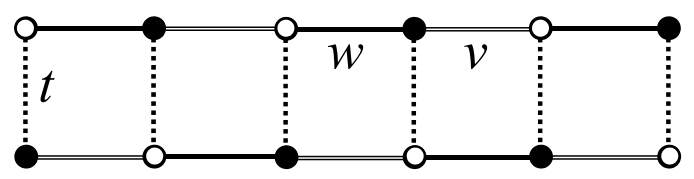

(b)

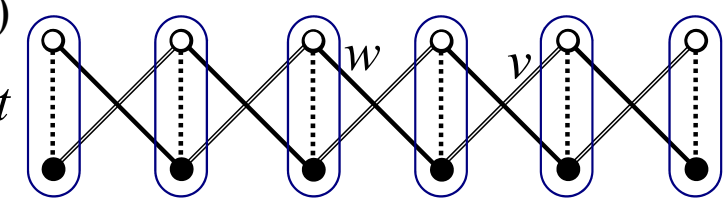

(c)

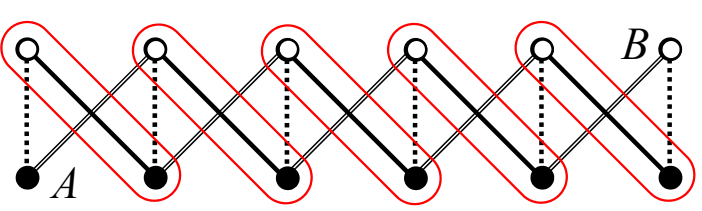

(d)

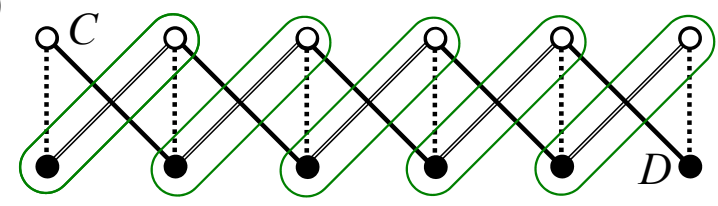

FIG. 1. (Color online) Schematics of the two coupled SSH chains and the formation of edge states. The system consists of two sublattices $A$ and $B$, indicated by filled and empty circles, respectively (a) Hopping amplitudes along each chain are staggered by $v$ (double line) and $w$ (single line). The interchain hopping amplitude is $t$ (dotted line). (b) Deformation of the system without changing the lattice structure. In this geometry a unit cell (circled by the blue line) contains two sites. In the case of $t \gg v, w>0$, the interchain dimerization (circled by the blue line) results in topologically trivial insulating phase, since there is no edge state. (c) In the limit $w \gg v, t>0$, edge states form at sites A and B. (d) Edge states form at sites $\mathrm{C}$ and $\mathrm{D}$ in the case $v \gg w, t>0$.

the transition between non-trivial and trivial topological phases, associated with the number of zero energy edge states as the topological invariant [27].

We consider a two coupled SSH chains system, which has a two-leg ladder structure. Fig. 1(a) sketches the geometry of the system, in which the hopping amplitudes in each leg is staggered and the rung indicates the interleg hopping. Such a ladder system is a bipartite lattice system, consisting of two sub-lattices $A$ and $B$. We write down the Hamiltonian for the system in a simple form

$$
H=\sum_{j=1}^{N}\left(w a_{j+1}^{\dagger} b_{j}+v a_{j}^{\dagger} b_{j+1}+t a_{j}^{\dagger} b_{j}\right)+\text { H.c. }
$$

where $a_{l}^{\dagger}$ and $b_{l}^{\dagger}$ are the creation operators of fermion at $l$ th site of sub-lattice $A$ and $B$, respectively. For a periodic boundary condition, which just like a ring, we take $a_{N+1}^{\dagger}=a_{1}^{\dagger}$ and $b_{N+1}^{\dagger}=b_{1}^{\dagger}$, while for an open one, we take
$a_{N+1}^{\dagger}=b_{N+1}^{\dagger}=0$. The ring situation can be a description for the bulk part of the system, i.e., the long central part of the open ladder. Taking the transformation

$$
\left\{\begin{array}{l}
a_{k}=\frac{1}{\sqrt{N}} \sum_{j} e^{i k j} a_{j} \\
b_{k}=\frac{1}{\sqrt{N}} \sum_{j} e^{i k j} b_{j}
\end{array},\right.
$$

we have

$$
H=\sum_{k} H_{k}=\sum_{k}\left(a_{k}^{\dagger}, b_{k}^{\dagger}\right) h_{k}\left(\begin{array}{c}
a_{k} \\
b_{k}
\end{array}\right)
$$

where

$$
h_{k}=\left(\begin{array}{cc}
0 & w e^{i k}+v e^{-i k}+t \\
w e^{-i k}+v e^{i k}+t & 0
\end{array}\right),
$$

and the wave vector $k=\pi(2 n-N) / N,(n=0,1, \ldots, N-$ 1). The Hamiltonian $H$ can be easily diagonalized since $\left[H_{k}, H_{k^{\prime}}\right]=0$. Therefore, the phase boundary can be obtained by the zero points of the spectrum

$$
\varepsilon_{k}= \pm \sqrt{\left|w e^{-i k}+v e^{i k}+t\right|^{2}} .
$$

From $\varepsilon_{k}=0$, we have equations

$$
\left\{\begin{array}{l}
(w+v) \cos k_{c}+t=0 \\
(w-v) \sin k_{c}=0
\end{array}\right.
$$

which determine the phase boundary and the position of band degeneracy point $k_{c}$. There are two types of boundaries, depending on the value of $k_{c}$. For $\sin k_{c} \neq 0$ we have $w=v$ and $\cos k_{c}=-t /(2 v)$. Then the first type of the boundary is

$$
w=v,|2 v / t|>1,
$$

which corresponds to two band degeneracy points $\pm\left|k_{c}\right|$. On the other hand, for $\sin k_{c}=0$, we have $\cos k_{c}=$ $-t /(w+v)$, it results in the second type of the boundary

$$
|(w+v) / t|=1,
$$

which corresponds to a single band degeneracy point $\pi$ or $-\pi$. We note that there are two triplet points at $w / t=$ $v / t= \pm 1 / 2$, which are joints connecting two types of boundary. We will see that such two types of boundaries correspond to topologically trivial and non-trivial states.

Now we focus on the quantum phases separated by the obtained boundaries. Matrix $h_{k}$ is the core of the Hamiltonian, containing all the information of the system. We rewrite $h_{k}$ as the form

$$
h_{k}=\mathbf{d}(k) \cdot \sigma
$$

with a 3 -D vector

$$
\left\{\begin{array}{l}
d_{x}(k)=(w+v) \cos k+t \\
d_{y}(k)=(v-w) \sin k \\
d_{z}(k)=0
\end{array}\right.
$$


where $\sigma$ represents 3-D Pauli matrix. The topology of the energy bands in each areas can be characterized by the loop of the curve [24]

$$
\left\{\begin{array}{l}
x=(w+v) \cos k+t, \\
y=(v-w) \sin k
\end{array}\right.
$$

in the auxiliary space $(x, y)$. The feature of the quantum phase is characterized by the topology of the loop. The winding number of the loop around the origin of $x y$ plane is defined as

$$
\mathcal{N}=\frac{1}{2 \pi} \int_{c} \frac{1}{r^{2}}(x \mathrm{~d} y-y \mathrm{~d} x)
$$

where $r^{2}=x^{2}+y^{2}$. A straightforward derivation from above definition yields

$$
\mathcal{N}=\left\{\begin{array}{cc}
0, & |t|>|w+v| \\
\operatorname{sgn}\left(v^{2}-w^{2}\right), & \text { otherwise }
\end{array},\right.
$$

where $\operatorname{sgn}($.$) denotes the sign function. It shows that$ there are three phases with $\mathcal{N}=0, \pm 1$, respectively. Actually, the conclusion of Eq. (13) can be obtained directly from the geometry of the loop represented by the parametric equations (11). Obviously, the loop is an ellipse with center located at $(t, 0)$ in the $x y$ plane, with the length of semiaxis along the $x$ (or $y$ ) axis is $|w+v|$ (or $|w-v|)$ (see Fig. 5). When $|t|>|w+v|$, the origin is out of the ellipse, resulting zero winding number. Otherwise, the winding number is \pm 1 . On the other hand, as $k$ increases from $-\pi$ to $\pi$, the rotating direction of the loop depends on the sign of the ratio $[(\partial x / \partial k) /(\partial y / \partial k)]_{k=\pi / 4}$ $=-(w+v) /(v-w)$, or the sign of $\left(v^{2}-w^{2}\right)$.

We plot the phase diagram in Fig. 2. According to the bulk-edge correspondence [22, 23], there should be gapless edge states when the bulk states are topologically nontrivial. Therefore, the quasi-zero modes should appear for finite open ladder when the parameters are taken in the region (I) and (III), but be absent in the region (II). To demonstrate this point, we plot the energy levels as functions of $v / t$ along the lines (a) $v / t-w / t=-1$, (b) $v / t-w / t=0$, (c) $v / t+w / t=2$, and (d) $v / t+w / t=0$, in the phase diagram, respectively. The plot in Fig. 3 are in agreement with the analysis, manifesting topological zero-energy modes for non-trivial areas.

We also plot the band structures presented by Eq. (5) for several typical points in different phases and at the phase boundary in Fig. 4 . We note that the zero points exhibit different geometries for different regions on the boundary. At boundaries between (I) and (III), there are always two zero points except for the triple points. In contrast, at boundaries between (II) and (I) or (III), there is always a single point. In the following section, we investigate this feature from the perspective of topology.

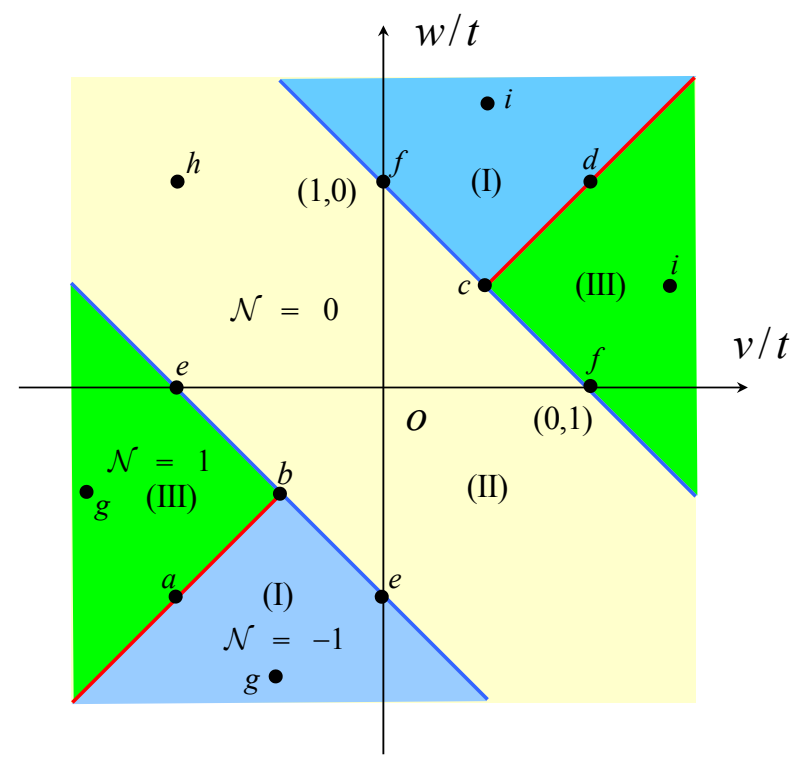

FIG. 2. (Color online) Phase diagram of the coupled SSH chains system on the $v w$ plane (in unit of t). The green line indicates the boundary separated the phases with Chern number $c= \pm 1$. The blue line indicates the boundary separated the phases with Chern number $|c|=1$ and 0 .

\section{TOPOLOGICAL FEATURE OF ZERO POINTS}

Now we focus on the state at the boundary of the system. From the analysis above, we know that there are two types of boundaries, which separate two quantum phases (I) and (III), (I) and (II) or (III) respectively. What makes this interesting is that for the first type of boundary, the winding number difference between two neighboring phases is 2 , while is 1 for the second type of boundary. This can be seen from the equation of the loop presented in Eq. (11). In general, the curve is an ellipse with center located at $(t, 0)$ in the $x y$ plane. For the first type of boundary, the curve reduces to

$$
\left\{\begin{array}{l}
x=2 v \cos k+t \\
y=0
\end{array}\right.
$$

which presents a segment of $x$ axis with length $4|v|$ and the center located at $(t, 0)$. Along the boundary, only the length of the segment varies. For the second type of boundary, the curve become

$$
\frac{(x-t)^{2}}{(w+v)^{2}}+\frac{y^{2}}{(w-v)^{2}}=1,
$$

which presents a normal ellipse passing through the origin $(0,0)$. Along the boundary, only the length of the semiaxis of the ellipse changes. To demonstrate this point, we plot graphs presented by Eq. (11) to schematically illustrate the difference between these two types of boundaries with trivial and non-trivial topologies. It indicates 

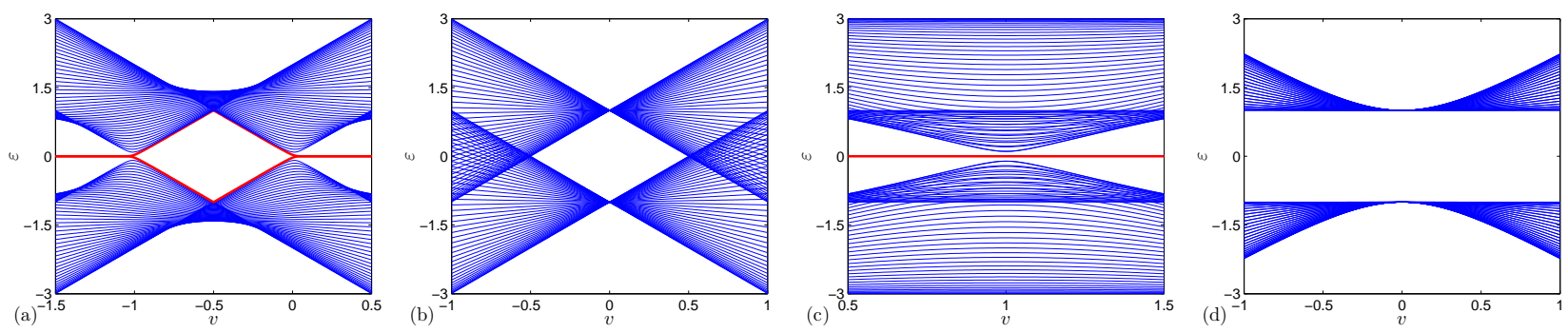

FIG. 3. (Color online) Energy spectra for the Hamiltonian in Eq. (1) as a function of $v / t$ on a $2 \times N$ ladder with the open boundary condition, obtained by exact diagonalization. The parameters obey the relations (a) $v / t-w / t=-1,(\mathrm{~b}) v / t-w / t=0$, (c) $v / t+w / t=2$, and (d) $v / t+w / t=0$. We see that energy gaps open in all three regions and the zero modes appear in the regions of (I) and (III) in the case of (a). The energy gap opens only in the region of (II) in the case of (b). The zero points in the regions of (I) and (III) are consisted of crossing points. In (c), there are always zero modes appear and the gap nearly close when arrive the point $v=w=1$. In (d), no zero modes appear all the time.
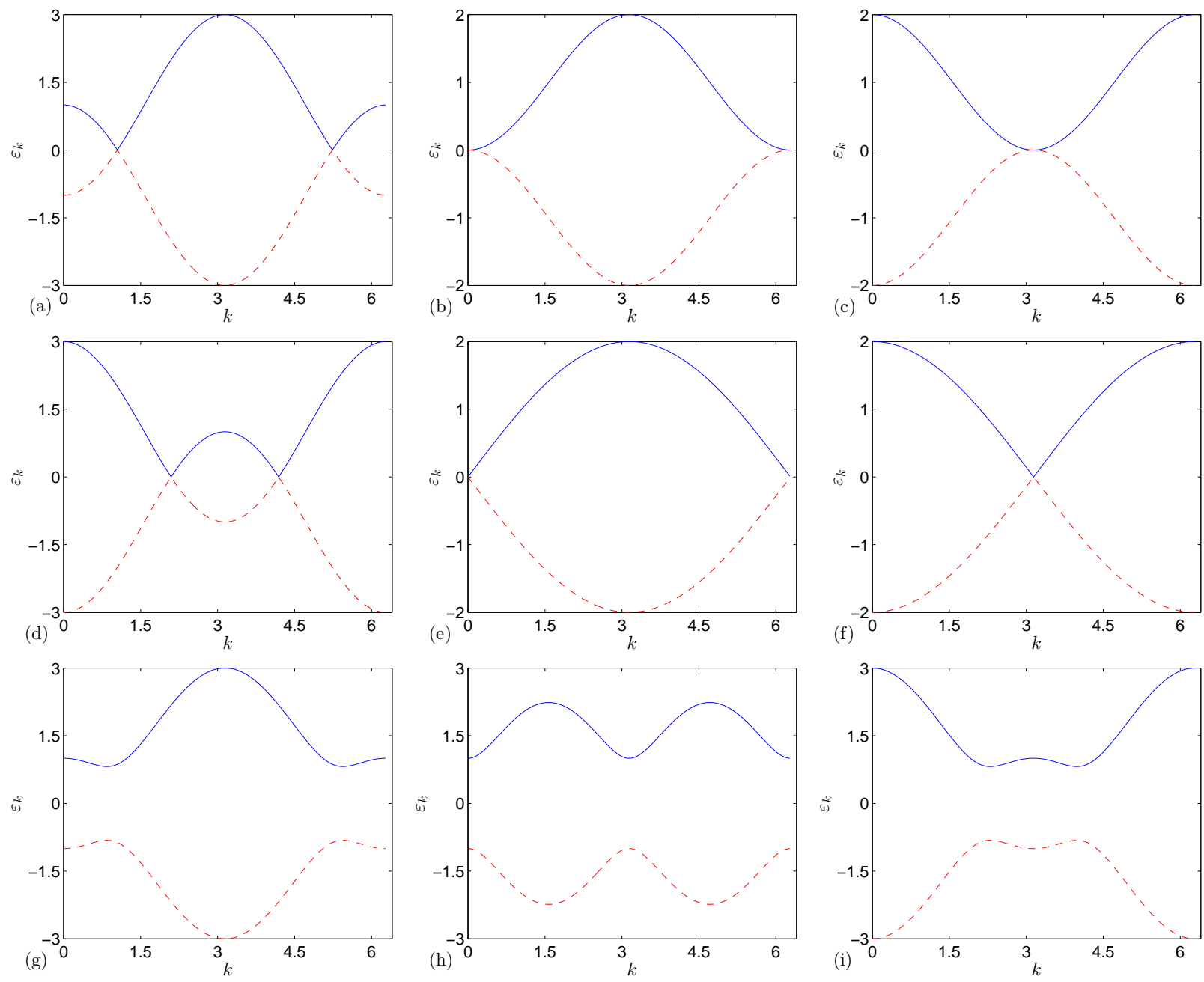

FIG. 4. (Color online) Plots of the structure of the energy band described by Eq. (5) at some points fixed in the Fig. 2 t $t=1$ for all figures. Graphs (a)-(f) just show the energy band situation in the phase boundaries $v=w$ and $v+w= \pm 1$. Meanwhile (g)-(i) display what energy band situation is in different phase areas. One can see that some symmetric points in Fig. 2 have the same band structure. 


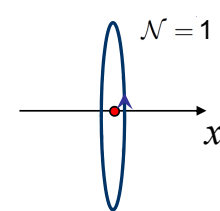

(a1)

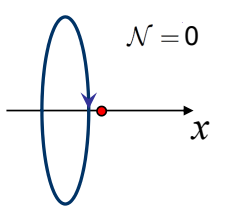

(a2)

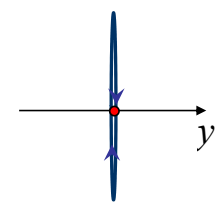

(b1)

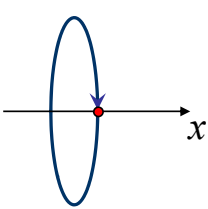

(b2)

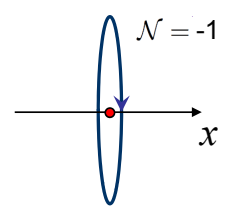

(c1)

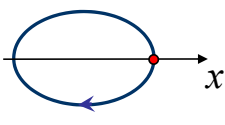

(c2)
FIG. 5. (Color online) Schematic illustration of two types of boundaries by the geometry of graphs in the auxiliary $x y$ plane. Red filled circle indicates the origin. Winding numbers and graphs of non-trivial topological phases with (a1) $v^{2}-w^{2}>0,(\mathrm{c} 1) v^{2}-w^{2}<0$. The non-trivial topological boundary with (b1) $w=v$, always corresponds to the graph of a segment. (a2) Graph and winding number of a trivial topological insulating phase. (b2) and (c2) Graphs of trivial topological boundary. They correspond to ellipses with various shapes, but passing through the origin. We see that two types of boundaries correspond to two completely different types of graphs.

that these two types of boundaries correspond to two completely different types of graphs. For the trivial one, the loop passes through the origin one time, while twice times for the non-trivial one, since the loop reduces to a segment.

In the following, we investigate the phase boundary from the other perspective. We introduce a 3-D vector field $\mathbf{F}(k)$ in $k$ space, which is defined as

$$
\mathbf{F}(k)=\left(\left\langle\sigma_{x}\right\rangle_{k},\left\langle\sigma_{y}\right\rangle_{k},\left\langle\sigma_{z}\right\rangle_{k}\right)
$$

where $\left\langle\sigma_{\alpha}\right\rangle_{k}=\left\langle k\left|\sigma_{\alpha}\right| k\right\rangle,(\alpha=x, y, z)$, the expectation value of $\sigma_{\alpha}$ for eigenstate $|k\rangle$ of $h_{k}$. In the case of $v=w$, we directly obtain

$$
\mathbf{F}(k)=(\operatorname{sgn}(2 v \cos k+t), 0,0)
$$

where $\operatorname{sgn}($.$) denotes the sign function. We note that the$ field $\mathbf{F}$ has a kink at the point $k_{c}=\cos [t /(2 v)]$, which is the gap closing point of the first type of the boundary. The topological charge of the kinks are 1 and -1 , respectively. In Fig. 6, we sketch the field $\mathbf{F}(k)$ to illustrate the kinks. Therefore, we conclude that the first type of nodal point is topologically protected. On the other hand, for second type of boundary with parameters satisfying $|(w+v) / t|=1$, we have

$$
\mathbf{F}(k)=(\operatorname{sgn}[t \pm(w+v)], 0,0),
$$

which has no kink in the $k$ space. In this case, the nodal point is topological trivial. (a)

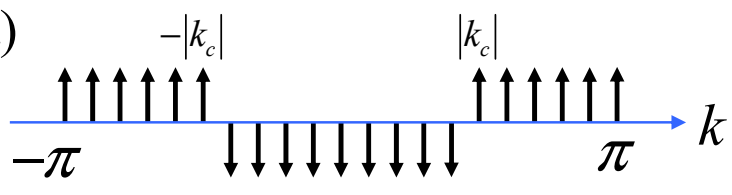

(b)

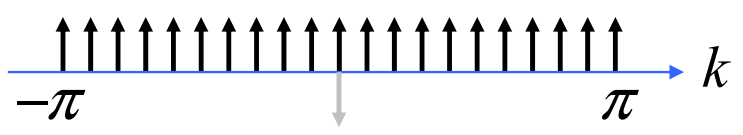

(c)

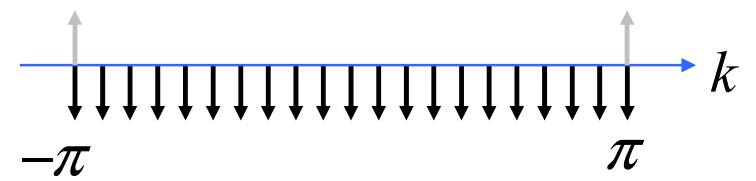

FIG. 6. (Color online) Schematics of topologically nontrivial field configurations. $\pm k_{c}$ denote the position of band degenerate points, which correspond to zero filled points. (a) System with $0<\left|k_{c}\right|<\pi$. There are two kinks at $\pm k_{c}$ with opposite topological charges. Two kinks can move towards or away from each other as parameters vary, but cannot be removed until the case (b) $k_{c}=0$, or (c) $\left|k_{c}\right|=\pi$. In (b) and (c), the flip of the vectors between black and gray arrows indicates the change of topology of the band degenerate point.

\section{PERTURBATIONS}

The topological invariant for the topological boundary is essentially the topologically unavoidable band touching points. It seems that there is nothing we can do to the system to get rid of the band touching point under the restriction in Eq. (77). Changing $w$ can only change the location of the band touching point. This topological feature may be robust for some kind of perturbation but fragile for others kinds. We consider two kinds of perturbations, with an extra diagonal hopping across two neighboring plaquettes and staggered on-site potential, respectively. Fig. 7 sketches the structures of two cases. We will focus on the effects of the extra terms on the topology of the boundary.

For the first case, the Hamiltonian can be written as

$$
H_{\mathrm{D}}=H+t_{\mathrm{D}} \sum_{j=1}^{N}\left(a_{j}^{\dagger} b_{j+2}+\text { H.c. }\right) \text {, }
$$

where $t_{\mathrm{D}}$ denotes the diagonal hopping amplitude. Based on the Fourier transformations in Eq. (2), we still have

$$
H_{\mathrm{D}}=\sum_{k}\left(a_{k}^{\dagger}, b_{k}^{\dagger}\right) h_{k}^{\mathrm{D}}\left(\begin{array}{l}
a_{k} \\
b_{k}
\end{array}\right),
$$


and

$$
h_{k}^{\mathrm{D}}=h_{k}+2 t_{\mathrm{D}} \cos 2 k\left(\begin{array}{cc}
0 & 1 \\
1 & 0
\end{array}\right) .
$$

The spectrum is

$$
\varepsilon_{k}^{\mathrm{D}}= \pm \sqrt{\left|w e^{-i k}+v e^{i k}+t+2 t_{\mathrm{D}} \cos (2 k)\right|^{2}},
$$

which only has a shift on $t$, i.e., $t \rightarrow t+2 t_{\mathrm{D}} \cos (2 k)$, from the spectrum $\varepsilon_{k}$ in Eq. (5). Thus the zero point can be obtained directly as following. Here we consider the case that the diagonal term is added as a perturbation, i.e., small $t_{\mathrm{D}}$ with $\left|t_{\mathrm{D}} / v\right| \ll 1$. For $\sin k_{c} \neq 0, \varepsilon_{k}^{\mathrm{D}}=0$ leads to $w=v$ and

$$
\cos k_{c} \approx-\frac{t}{2 v}\left(1+\frac{t_{\mathrm{D}} t}{v^{2}}\right)
$$

which identifies the boundary line

$$
w=v,\left|\frac{t}{2 v}\left(1+\frac{t_{\mathrm{D}} t}{v^{2}}\right)\right|<1 .
$$

On the other hand, for $\sin k_{c}=0$, the boundary is the line

$$
|w+v|=\left|t+t_{\mathrm{D}}\right| .
$$

We note that for small $t_{\mathrm{D}}$, the phase diagram changes a little comparing to the case with zero $t_{\mathrm{D}}$, but keeping the original geometry. The position of the kink, $k_{c}$ shifts a little, without changing the original topology, i.e., the topological charge of the kink. Then the gapless state is topologically invariant under the perturbation from the $t_{\mathrm{D}}$-term.

For the second case, the Hamiltonian can be written as

$$
H_{\mathrm{V}}=H+V \sum_{j=1}^{N}\left(b_{j}^{\dagger} b_{j}-a_{j}^{\dagger} a_{j}\right)
$$

which indicates that particles on different sub-lattices have opposite chemical potentials. The extra potentials do not break the translational symmetry. By the same procedure, we have

$$
H_{\mathrm{V}}=\sum_{k}\left(a_{k}^{\dagger}, b_{k}^{\dagger}\right) h_{k}^{\mathrm{v}}\left(\begin{array}{c}
a_{k} \\
b_{k}
\end{array}\right),
$$

and

$$
h_{k}^{\mathrm{v}}=\left(\begin{array}{cc}
-V & w e^{i k}+v e^{-i k}+t \\
w e^{-i k}+v e^{i k}+t & V
\end{array}\right) .
$$

The spectrum is

$$
\varepsilon_{k}^{\mathrm{v}}= \pm \sqrt{\left|w e^{-i k}+v e^{i k}+t\right|^{2}+V^{2}},
$$

(a)

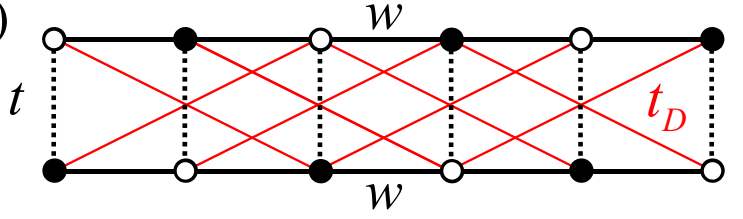

(b)

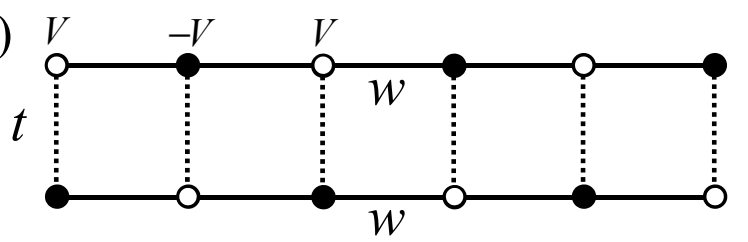

(c)

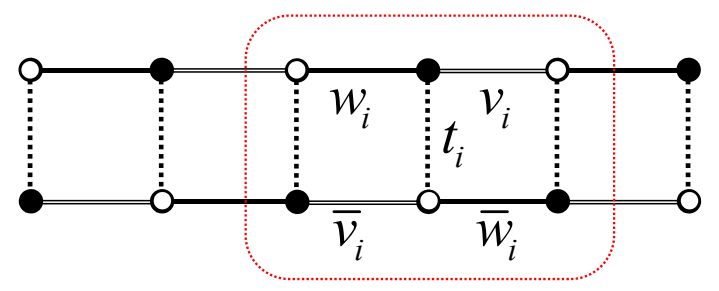

FIG. 7. (Color online) Schematics of two kinds of perturbation on the coupled SSH chain at semi-metal point. (a) Diagonal hopping term (red line) with amplitude $t_{D}$ across two neighboring plaquettes. (b) Staggered on-site potentials on two sublattices indicated by filled and empty circles, respectively. In the case of (a), the topology of zero-energy points is invariant under the perturbations, while the gap opens for non-zero $V$ in the case (b). (c) Schematic illustration of the mechanism of degenerate point which is irrelevant to the symmetry of the system. The ladder system is consisted of double plaquette (circled by the red dotted line). The existence of zero-energy degenerate point requires the balance of the local parameters $\left\{w_{i}, v_{i}, \bar{w}_{i}, \bar{v}_{i}, t_{i}\right\}$, presented in Eq. (49).

which clearly shows that the nonzero $V$ can open a gap, destroying the topological gapless state. In the vicinity of $k_{c}$, we have

$$
\left(\frac{\partial\left|\varepsilon_{k}^{\mathrm{v}}\right|}{\partial V}\right)_{k \approx k_{c}} \approx \frac{V}{|V|},
$$

which is discontinuous at the point $V=0$. It is a critical point of second-order quantum phase transition, which is associated with inversion symmetry (exchange $A$ and $B$ ) breaking. In fact, condition $w=v$ takes the role of maintaining the inversion symmetry.

\section{SYMMETRY PROTECTION OF KINKS}

The kinks, as a topological invariant, should be protected by the certain symmetry of the system. In the following, we investigate the symmetry associated with the topological nodal points. The Hamiltonian possesses many symmetries, for instance, translational symmetry, time reversal symmetry, etc.. For $w=v$, the system reduces to a ladder with identical legs. There are two 
specific symmetries related to the translational operator $\hat{T}_{1}$ and inversion operator $\hat{P}$, which are defined as

$$
\hat{T}_{1} a_{j} \hat{T}_{1}^{-1}=b_{j+1}, \hat{T}_{1} b_{j} \hat{T}_{1}^{-1}=a_{j+1},
$$

and

$$
\hat{P} a_{j} \hat{P}^{-1}=b_{j}, \hat{P} b_{j} \hat{P}^{-1}=a_{j} .
$$

The action of $\hat{T}_{1}$ shifts a lattice space along the legs of the ladder in Fig. 1(a). In general, $\hat{T}_{1}$ does not commute with the Hamiltonian with $w \neq v$ but $\hat{T}_{1}^{2}$ does. In parallel, the action of $\hat{P}$ exchange two sublattices $A$ and $B$, or two legs of the ladder in Fig. 1(a). In general, $\hat{P}$ does not commute with the Hamiltonian except for the case $w=v$. Then in the case of $w=v$, we have

$$
\left[H, \hat{T}_{1}\right]=[H, \hat{P}]=0 .
$$

Operators $H, \hat{T}_{1}$, and $\hat{P}$ have common eigenstate set, which can be obtained as

$$
\left|\psi_{k}^{ \pm}\right\rangle=\frac{1}{\sqrt{2 N}} \sum_{j} e^{-i k j}\left(|j\rangle_{b} \pm|j\rangle_{a}\right),
$$

with the eigen energy

$$
\varepsilon_{k}^{ \pm}= \pm|2 v \cos k+t|
$$

in the single-particle invariant subspace spanned by basis $|j\rangle_{a}=a_{j}^{\dagger}|0\rangle$ and $|j\rangle_{b}=b_{j}^{\dagger}|0\rangle$. In general, these eigenstates satisfy

$$
\hat{T}_{1}\left|\psi_{k}^{ \pm}\right\rangle= \pm e^{i k}\left|\psi_{k}^{ \pm}\right\rangle
$$

and

$$
\hat{P}\left|\psi_{k}^{ \pm}\right\rangle= \pm\left|\psi_{k}^{ \pm}\right\rangle .
$$

Nevertheless, the two-fold zero-energy degenerate states (with the wave vector $k_{c}$ ) of $H$ can be expressed as

$$
\left|\psi_{k_{c}}^{a, b}\right\rangle=\frac{1}{\sqrt{N}} \sum_{j} e^{-i k_{c} j}|j\rangle_{a, b},
$$

which satisfy

$$
\hat{T}_{1}\left|\psi_{k_{c}}^{a}\right\rangle=e^{i k_{c}}\left|\psi_{k_{c}}^{b}\right\rangle, \hat{T}_{1}\left|\psi_{k_{c}}^{b}\right\rangle=e^{i k_{c}}\left|\psi_{k_{c}}^{a}\right\rangle,
$$

and

$$
\hat{P}\left|\psi_{k_{c}}^{a}\right\rangle=\left|\psi_{k_{c}}^{b}\right\rangle, \hat{P}\left|\psi_{k_{c}}^{b}\right\rangle=\left|\psi_{k_{c}}^{a}\right\rangle .
$$

This indicates that the nodal points at $k_{c}$ are protected by the symmetry related to $\hat{T}_{1}$ or $\hat{P}$.

Furthermore, when we consider the half-filled case, the topological nodal point is connected to a QPT associated with spontaneously symmetry breaking. We consider the phase diagram along the axis $w=v$, which is sketched in
Fig. 8. A second order QPT occurs at $v= \pm t / 2$, since the first order derivative of groundstate energy with the respect to $v$ is nonanalytic at the two points. This can be seen from the fact that $\varepsilon_{k}^{-}$is nonanalytic at point $k=k_{c}$. In the region of $|v / t|<1 / 2$, the gapped ground state can be expressed as

$$
|G\rangle=\prod_{k}\left|\psi_{k}^{-}\right\rangle
$$

which is singlet. In the region of $|v / t|>1 / 2$, the ground states are gapless and have the form

$$
\left|G_{a, b}\right\rangle=\left|\psi_{k_{c}}^{a, b}\right\rangle \prod_{k \notin k_{c}}\left|\psi_{k}^{-}\right\rangle
$$

which are double degenerate. Since

$$
\hat{T}_{1}\left|G_{a}\right\rangle=-\left|G_{a}\right\rangle, \hat{T}_{1}\left|G_{b}\right\rangle=-\left|G_{a}\right\rangle,
$$

and

$$
\hat{P}\left|\psi_{k_{c}}^{a}\right\rangle=-\left|\psi_{k_{c}}^{b}\right\rangle, \hat{P}\left|\psi_{k_{c}}^{b}\right\rangle=-\left|\psi_{k_{c}}^{a}\right\rangle,
$$

the translational $\hat{T}_{1}$ and inversion $\hat{P}$ symmetries are spontaneously broken. Remarkably, the doubly degenerate ground states are topologically protected by the symmetries of translation $\hat{T}_{1}$ and inversion $\hat{P}$. Thus, we come to the conclusion that this model provides an example to connect the topological QPT and the one associated with spontaneous breaking, which has been studied previously in the quantum spin system [24].

On the other hand, in the case of $|(w+v) / t|=1$, the two-fold zero-energy degenerate states are the form

$$
\left|\psi_{ \pm}^{a, b}\right\rangle=\frac{1}{\sqrt{N}} \sum_{j}( \pm 1)^{j}|j\rangle_{a, b},
$$

which satisfy

$$
\hat{T}_{1}\left|\psi_{ \pm}^{a}\right\rangle= \pm\left|\psi_{ \pm}^{b}\right\rangle, \hat{T}_{1}\left|\psi_{ \pm}^{b}\right\rangle= \pm\left|\psi_{ \pm}^{a}\right\rangle,
$$

and

$$
\hat{P}\left|\psi_{ \pm}^{a}\right\rangle=\left|\psi_{ \pm}^{b}\right\rangle .
$$

Although $\left|\psi_{ \pm}^{a, b}\right\rangle$ and $\left|\psi_{k_{c}}^{a, b}\right\rangle$ have the similar properties, in this situation, we have

$$
\left[H, \hat{T}_{1}\right] \neq 0,[H, \hat{P}] \neq 0 .
$$

The nodal point is irrelevant to the symmetry of the system. It may be due to the mechanism of this type of degeneracy. We note that the key point of the degeneracy arises from the balance of local parameters. As illustrated in Fig. 7(c), a general ladder is consisted of many double plaquettes. The existence of degeneracy requires

$$
\left|w_{i}+v_{i}\right|=\left|\bar{w}_{i}+\bar{v}_{i}\right|=\left|t_{i}\right|
$$




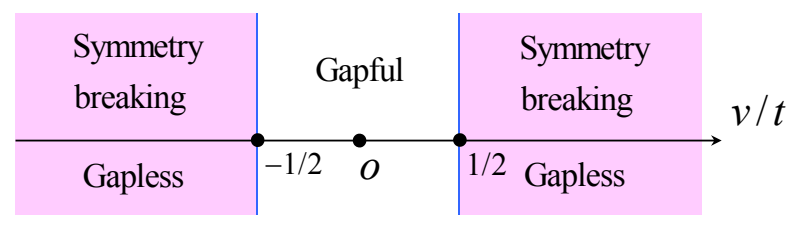

FIG. 8. (Color online) Schematic phase diagram of ground state of half-filled spinless fermionic system along the axis of $w=v$. In the region of $|v / t|>1$, the translational $T_{1}$ and inversion $P$ symmetries are spontaneously broken, associated with gapless ground state. In the region of $|v / t|<1$, the ground state is gapful and possesses the translational $T_{1}$ and inversion $P$ symmetries.

for every double plaquette indexed by $i$. There should be infinite sets of parameters $\left\{w_{i}, v_{i}, \bar{w}_{i}, \bar{v}_{i}, t_{i}\right\}$ satisfy the equations. In this sense, such degeneracy is accident and irrelevant to the symmetry of the system.

We close this section by noting that the operator $\hat{T}_{1}$ and $\hat{P}$ are not antiunitary operators. This differs from the one appears in higher dimensional system [11]. It turns out that the time-reversal symmetry takes a central role contributing to the protection of degeneracies in 2-D systems. In contrast, the model we study is a quasi1-D and possesses time-reversal symmetry, referring to nonmagnetic material.

\section{SUMMARY}

In summary, we studied two coupled SSH chains system, which possesses a little more complicated phase diagram in comparison with that of SSH chain. It contains three phases, two non-trivial topological insulating phases and one trivial phase. Moreover, the boundary between these two quantum phases is still topologically nontrivial, which arises from two topologically unavoidable band closing points. As inter-chain coupling strength varies, two topological nodal points appear, move, merge and disappear in $k$ space. We also show that the topological invariant of the boundary is protected by the translational and inversion symmetries, rather than the antiunitary operation in the case of 2-D semimetal phase. Based on the results, we established the possible connection between the second order QPT, associated with a gap closing and spontaneously symmetry breaking, and the topological QPT characterized by the change of topological invariant. Our finding extends the understanding of topological gapless phase and provides a platform to experimentally work within the simple system, which has particular advantages: a lower dimension and without the need of magnetic flux.

\section{ACKNOWLEDGMENTS}

We acknowledge the support of CNSF (Grant No. 11374163).
[1] X. Wan, A. M. Turner, A. Vishwanath, and S. Y. Savrasov, Phys. Rev. B 83, 205101 (2011).

[2] K. Y. Yang, Y. M. Lu, and Y. Ran, Phys. Rev. B 84, 075129 (2011).

[3] A. A. Burkov and L. Balents, Phys. Rev. Lett. 107, 127205 (2011).

[4] G. Xu, H. Weng, Z. Wang, X. Dai, and Z. Fang, Phys. Rev. Lett. 107, 186806 (2011).

[5] W. W. Krempa and Y. B. Kim, Phys. Rev. B 85, 045124 (2012).

[6] H. Weng, C. Fang, Z. Fang, B. A. Bernevig, and X. Dai, Phys. Rev. X 5, 011029 (2015).

[7] S. M. Huang, et al. Nat. Commun. 6, 7373 (2015).

[8] S. M. Young, et al. Phys. Rev. Lett. 108, 140405 (2012).

[9] Z. Wang, et al. Phys. Rev. B 85, 195320 (2012).

[10] Z. Wang, H. Weng, Q. Wu, X. Dai, and Z. Fang, Phys. Rev. B 88, 125427 (2013).

[11] J. M. Hou, Phys. Rev. Lett. 111, 130403 (2013); J. M. Hou, Phys. Rev. B 89, 235405 (2014); J. M. Hou and W. Chen, Sci. Rep. 5, 17571 (2015); J. M. Hou and W. Chen, Sci. Rep. 6, 33512 (2016).

[12] D. Sama, Nature. Phys. 8, 67-70 (2012).

[13] Z. K. Liu, et al. Science 343, 864-867 (2014).

[14] M. Neupane, et al. Nat. Commun. 5, 3786 (2014).
[15] S. Y. Xu, et al. Science 349, 613-617 (2015).

[16] B. Q. Lv, et al. Phys. Rev. X 5, 031013 (2015).

[17] L. Lu, et al. Science 349, 622-624 (2015).

[18] R. T. Clay and S. Mazumdar, Phys. Rev. Lett. 94, 207206 (2005).

[19] Y. Shimizu, S. Aoyama, T. Jinno, M. Itoh, and Y. Ueda, Phys. Rev. Lett. 114, 166403 (2015).

[20] T. Zhang and G. B. Jo, Sci. Rep. 5, 16044 (2015).

[21] S. Sachdev, Quantum Phase Transitions (Cambridge University Press, Cambridge, 1999).

[22] M. Z. Hasan and C. L. Kane, Rev. Mod. Phys. 82, 3045 (2010).

[23] X. L. Qi and S. C. Zhang, Rev. Mod. Phys. 83, 1057 (2011).

[24] G. Zhang and Z. Song, Phys. Rev. Lett. 115, 177204, (2015).

[25] W. P. Su, J. R. Schrieffer, and A. J. Heeger, Phys. Rev. Lett. 42, 1698 (1979).

[26] J. Zak, Phys. Rev. Lett. 62, 2747 (1989).

[27] J. K. Asbóth, L. Oroszlány, and A. Pályi, A Short Course on Topological Insulators: Band Structure and Edge States in One and Two Dimensions, Lecture Notes in Physics (Springer International Publishing, Switzerland, 2016). 